\title{
Differential Frost Tolerance and Enzymatic Activities in the Leaves and Immature Fruits of Loquat (Eriobotrya japonica Lindl.)
}

\author{
Guohua Zheng ${ }^{1}$, Xianqian Niu², Jinbiao Zhang ${ }^{3}$, Hanwen $\mathrm{Wu}^{4}$, Xiuxiang $\mathrm{Lin}^{2}$, and Dongming $\mathrm{Pan}^{1 *}$ \\ ${ }^{1}$ Horticulture College, Fujian Agriculture and Forestry University, Fuzhou 350002, China \\ ${ }^{2}$ Fujian Science Institute of Tropical Crops, Zhangzhou 363001, China \\ ${ }^{3}$ College of Life Science, Fujian Agriculture and Forestry University, Fuzhou 350002, China \\ ${ }^{4}$ Graham Centre for Agricultural Innovation (NSW Department of Primary Industries and Charles Sturt University), Wagga Wagga Agricultural Institute, \\ PMB, Wagga Wagga NSW 2650, Australia
}

\begin{abstract}
In this experiment, five commercial cultivars and one wild species of loquat were used to investigate frost tolerance and enzymatic activities in leaves and young fruits under cold stress at $-3^{\circ} \mathrm{C}$. The frost injury, malondialdehyde (MDA) content, and oxygen-scavenging enzyme activities of superoxide dismutase (SOD), peroxidase (POD), catalase (CAT) and ascorbate peroxidase (APX) were studied. This results showed that the wild species 'Wild Oak-leaf' loquat was the most frost tolerant among accessions tested, followed by the cultivar 'Golden Block'. Other cultivars, 'Wu Gong Bai', 'Taicheng 4', 'Xiangzhong 11' and 'Zaozhong 6', were relatively weak in frost tolerance. The enzymatic activities of SOD, POD and CAT increased initially and then decreased as the exposure time increased. However, the enzymatic peak occurred later in the frost-tolerant accession than in the frost-sensitive accession. The correlation coefficients of MDA contents between leaves and immature fruits were from 0.93 to 0.99 in the five commercial loquat cultivars. For the 'Wild Oak-leaf' loquat, the correlation coefficients of MDA and POD were 0.98 and 0.95, respectively, but the coefficients for SOD, CAT and APX were relatively low. In general, there were good correlations between loquat leaves and immature fruits in MDA content and enzyme activities. These results indicate that analysis of these physiological and biochemical activities in loquat leaves could potentially be used to predict the cold tolerance in loquat at immature fruit stage and to accelerate breeding programs for cold tolerance in loquat.
\end{abstract}

Additional key words: cold treatment, frost-tolerance, MDA, oxygen-scavenging enzymes

\section{Introduction}

Loquat (Eriobotrya japonica Lindl.) originated from China and is one of the favorite sub-tropical fruits in spring when there are limited fresh fruits available. It has been introduced to many oversea countries, such as Japan, India, Australia, New Zealand, Madagascar, South Africa, and Mediterranean countries (He et al., 2011). The fresh fruits of loquat can be consumed directly or processed into peeled can, jam, and jelly. Its leaves have been documented to possess medicinal values and have been used to treat skin diseases, diabetes, chronic bronchitis, coughs, phlegm, and ulcers (Ferreres et al., 2009). China is the world's largest producer of loquat with a cultivation area of 120,000 ha, producing more than $400,000 \mathrm{t}$ of fresh fruit per year (Hueso and Cuevas, 2008). Putian city of Fujian province is one of the largest loquat production regions in China. The annual production is over 80,000 t (Jiang et al., 2010).

Loquat usually blossoms in autumn and winter, and sets fruits in spring. So the flowers and early fertilized young fruit are at a high risk of frost damage. Generally, the frost damage to loquat occurs when temperature drops to less than $-6^{\circ} \mathrm{C}$ at flowering stage and to less than $-3^{\circ} \mathrm{C}$ at the early fruiting stage (Yang et al., 2003). Jiang et al. (2010) reported that more than $80 \%$ loquat trees in Putian regions were affected by the extreme cold weather in 2004,

\footnotetext{
*Comesponding author: pdm666@126.com

※ Received 29 April 2014; Revised 9 December 2014; Accepted 21 December 2014.

(C) 2015 Korean Society for Horticultural Science
} 
causing yield reduction of 13,300 $t$ and economic loss of 65 million Chinese yuan. Frost damage is therefore a major limiting factor for loquat production and area expansion (Wang et al., 2008). Selecting strongly frost-tolerant accessions was the most effective way to solve this problem.

It has been reported that some physiological indicators could be used to assist the selection of accessions with elevated level of frost tolerance. For example, Wang et al. (1999) found that malondialdehyde (MDA) content increased as the temperature decreased and it also corresponded well with the changes in membrane permeability in apricot (Armeniaca vulgaris Lam.), suggesting that MDA content could be used as a physiological indicator to identify frosttolerant fruit accessions. Studies have also shown that the activities of some oxygen-scavenging enzymes such as superoxide superoxide dismutase (SOD), catalase (CAT), peroxidase (POD) and ascorbate peroxidase (APX) were closely related to the frost-tolerance in cucumber (Cucumis sativus L.) (Liu et al., 1985), pepper (Capsicum annuum L.) (Zhou and Lu, 1994), tomato (Lycopersicon esculentum) (Ren et al., 2002), citrus (Citrus L.) (Peng and Sun, 2000), fig (Ficus carica L.) (Jiang et al., 1992), apricot (A. vulgaris Lam.) (Wang et al., 1999) and banana (Musa spp.) (Zhou et al., 1999), and the enzymatic activities was higher in frost-tolerant accessions than in the frost-sensitive accessions. However, limited information is available about MDA and oxygen-scavenging enzymes and their association with frost tolerance in loquat (Sun et al., 2011).

The early fruit development stage was the most sensitive to frost damage. Loquat flowering and early fruit development often coincides with freezing temperature between December and January, which is a major bottleneck in breeding frosttolerant cultivars. The identification of frost tolerant loquat accessions was traditionally based on some physiological indicators, such as SOD and MDA of the young fruits. Up to now, there are little physiological studies on the relationship in frost tolerance between leaves and young fruits in loquat. The aim of this study was to determine the relationship in MDA, SOD, POD, CAT, and APX between leaves and fruits in loquat accessions with varying levels of frost tolerance. Such relationship could greatly accelerate the breeding programs for frost-tolerant accessions.

\section{Materials and Methods}

\section{Fruit tree materials}

In January 2007, scions of loquat (Eriobotrya japonica) cultivars 'Wu Gong Bai', 'Zaozhong 6', 'Taicheng 4', 'Xiangzhong 11', 'Golden Block' and one wild species 'Wild Oak-leaf' loquat (Eriobotrya prinoides Rehd) were collected from the National Germplasm Nursery, Fujian Academy of Agriculture Sciences, China. The scions were grafted onto the rootstock of Jiefangzhong and grown in pots (one plant per pot) in an orchard in Fujian Agricultural and Forestry University, Fujian, China. The grafted trees were placed randomly, irrigated and fertilized according to common district practices. Three years after the grafting, trees with uniform and vigorous growth were selected and used as experimental materials.

\section{Cold treatment}

The cold stress experiment was carried out in the Horticultural Laboratory of Fujian Agriculture and Forestry University from March to April, 2009. The cold treatment procedure was adopted from the method of Xie and $\mathrm{Li}$ (2006) and Wang et al. (2008). The treatment temperature was set at $-3^{\circ} \mathrm{C}$ and a gradient cooling method using a temperature controllable freezer (ETC-100, Shanghai Jingchuang Electronics Manufacturing Com., Ltd. China). The temperature was initially set to $10^{\circ} \mathrm{C}$ and gradually decreased to $4^{\circ} \mathrm{C}$ at a rate of $1^{\circ} \mathrm{C} / \mathrm{h}$ and then remained constant at $4^{\circ} \mathrm{C}$ for $10 \mathrm{~h}$. The temperature was further decreased to $-3^{\circ} \mathrm{C}$ at a rate of $2^{\circ} \mathrm{C} / \mathrm{h}$. The forth and fifth leaves of loquats and healthy immature young fruits were sampled for the analyses of MDA, POD, SOD, CAT, and APX at 0, 1.5, 3, $4.5,6$, and $7.5 \mathrm{~h}$ after cold treatment at $-3^{\circ} \mathrm{C}$. The materials sampled after different cold exposure periods were cut into small pieces, well mixed and treated with liquid nitrogen, and then stored at $-40^{\circ} \mathrm{C}$ deep freezer. The treated samples were thawed under ambient temperature. Leaf or fruit browning was observed after $12 \mathrm{~h}$. The leaf with more than $50 \%$ browning in total leaf area was considered as cold-damaged leaf. The fruit flesh which turned dark brown were considered as cold damage (Wang et al., 2008).

\section{Determination of MDA content}

Lipid peroxidation was estimated by the level of MDA. The MDA content of loquat leaves was determined according to the modified method of Liu and Zhang (1994). One gram of frozen material for each sample was ground in $8 \mathrm{~mL}$ of $0.05 \mathrm{~mol} \cdot \mathrm{L}^{-1}$ ice-cold phosphate buffer solution (PBS, pH 7.8). The homogenate was centrifuged at 15,000 $\times \mathrm{g}$ for $30 \mathrm{~min}$ at $4^{\circ} \mathrm{C}$. A reaction was carried out by mixing $2 \mathrm{~mL}$ of sample supernatant with $2 \mathrm{~mL}$ of $0.6 \%$ thiobarbituric acid containing 10\% trichloroacetic acid (TCA) and placing it on a temperature controlled bath at $100^{\circ} \mathrm{C}$ for $15 \mathrm{~min}$. The reaction solution was then quickly cooled on ice. After centrifugation at $15,000 \times \mathrm{g}$ for $15 \mathrm{~min}$ at $4^{\circ} \mathrm{C}$, the supernatant 
was collected and its absorbance was measured at $450 \mathrm{~nm}$, $532 \mathrm{~nm}$, and $600 \mathrm{~nm}$ respectively on a Libra S22 UV-visible spectrophotometer (Biochrom, UK). The concentration of MDA in reaction solution was expressed as $\mu \mathrm{mol} \cdot \mathrm{L}^{-1}$ and calculated as follow:

$$
\operatorname{MDA}\left(\mu \mathrm{mol} \cdot \mathrm{L}^{-1}\right)=6.45 \times\left(\mathrm{A}_{532 \mathrm{~nm}}-\mathrm{A}_{600 \mathrm{~nm}}\right)-0.56 \times \mathrm{A}_{450 \mathrm{~nm}}
$$

\section{Determination of POD and SOD activities}

The frozen leaf or young fruit material $(0.2 \mathrm{~g})$ was homogenized with a mortar and pestle on ice in $7 \mathrm{~mL}$ of 50 $\mathrm{mmol} \cdot \mathrm{L}^{-1}$ chilled Tris- $\mathrm{HCl}(\mathrm{pH} 7.0$ ) containing $20 \%$ glycerol, $1 \mathrm{mmol} \cdot \mathrm{L}^{-1}$ ascorbic acid (AsA), $1 \mathrm{mmol} \cdot \mathrm{L}^{-1}$ dithiothreitol (DTT), $1 \mathrm{mmol} \cdot \mathrm{L}^{-1}$ reduced glutathione $(\mathrm{GSH})$, and $5 \mathrm{mmol} \cdot \mathrm{L}^{-1}$ $\mathrm{MgCl}_{2}$. The homogenate was centrifuged at $25,000 \times \mathrm{g}$ for $30 \mathrm{~min}$ at $4^{\circ} \mathrm{C}$ and the resulting supernatant was used for enzyme assay. POD activity was determined according to the method of Giannopolitics and Ries (1977). The reaction mixture consisted of $0.019 \mathrm{~mL}$ guaiacol, $0.028 \mathrm{~mL} \mathrm{H}_{2} \mathrm{O}_{2}$ (34\%) and $50 \mathrm{~mL}$ of $200 \mathrm{mmol} \cdot \mathrm{L}^{-1}$ phosphate buffer solution (PBS, pH 6.0). The absorbance was measured at $470 \mathrm{~nm}$ after adding $10 \mu \mathrm{L}$ of the supernatant collected above into a test tube containing $3 \mathrm{~mL}$ reaction mixture for $5 \mathrm{~min}$. SOD activity was assayed using the method of Zhu (1990) with minor modification. The reaction medium consisted of $50 \mathrm{mmol} \cdot \mathrm{L}^{-1} \mathrm{PBS}(\mathrm{pH} 8), 0.1 \mathrm{mmol} \cdot \mathrm{L}^{-1} \mathrm{EDTA}, 13.37$ $\mathrm{mmol} \cdot \mathrm{L}^{-1} \mathrm{l}$-methionine (Met), and $77.12 \mu \mathrm{mol} \cdot \mathrm{L}^{-1}$ nitroblue tetrazolium chloride (NBT). The mixture contained $3.86 \mathrm{~mL}$ reaction medium, $100 \mu \mathrm{L}$ riboflavin $\left(80.2 \mu \mathrm{mol} \cdot \mathrm{L}^{-1}\right)$, and $50 \mu \mathrm{L}$ enzyme extract was kept under 4000 Lux fluorescent light for $30 \mathrm{~min}$. The changes of absorbance were measured at $560 \mathrm{~nm}$.

\section{Determination of CAT and APX activities}

The frozen leaf or young fruit material $(0.2 \mathrm{~g})$ was homogenized in $7 \mathrm{~mL}$ of $50 \mathrm{mmol} \cdot \mathrm{L}^{-1}$ chilled PBS (pH 7.6) containing $0.1 \mathrm{mmol} \cdot \mathrm{L}^{-1}$ EDTA and $0.5 \%$ Triton X-100 and then centrifuged at $25,000 \times \mathrm{g}$ for $30 \mathrm{~min}$ at $4^{\circ} \mathrm{C}$. CAT activity was measured using the method of Rao et al. (1996) with slight modification. Briefly, $1 \mathrm{~mL}$ reaction mixture contained $100 \mu \mathrm{L}$ PBS $\left(100 \mathrm{mmol} \cdot \mathrm{L}^{-1}, \mathrm{pH} 7.0\right)$ and $20 \mu \mathrm{L}$ supernatant. The reaction was initiated by the addition of $10 \mu \mathrm{L}$ of $10 \% \mathrm{H}_{2} \mathrm{O}_{2}$. The absorbance was measured at $240 \mathrm{~nm}$. APX activity was determined based on the method of Chen and Cheng (2003) with some modification. The reaction mixture $(1 \mathrm{~mL})$ contained $100 \mu \mathrm{L}$ of $500 \mathrm{mmol} \cdot \mathrm{L}^{-1}$ PBS (pH7.5), $2 \mu \mathrm{L}$ of $50 \mathrm{mmol} \cdot \mathrm{L}^{-1} \mathrm{EDTA}, 10 \mu \mathrm{L}$ of $50 \mathrm{mmol} \cdot \mathrm{L}^{-1}$ AsA, and $50 \mu \mathrm{L}$ supernatant. The reaction was initiated by the addition of $20 \mu \mathrm{L}$ of $10 \% \mathrm{H}_{2} \mathrm{O}_{2}$. The activity was calculated from the change in absorbance measured at $290 \mathrm{~nm}$.

\section{Statistical analysis}

This study used a randomised complete block design with six loquat accessions, six cold treatment periods of $0,1.5,3,4.5,6$, and $7.5 \mathrm{~h}$ at $-3^{\circ} \mathrm{C}$ and three replicates. Each replicate consisted of one loquat tree, resulting in a total of 108 loquat trees used in this experiment. The mean values of three replicates were presented. Statistical analyses were carried out by DPS using Tukey-test for the different significance test.

\section{Results}

\section{Changes in browning rate under cold stress}

The dynamic changes of browning rate over time under low temperature stress followed the sigmoidal responses both in the leaves and fruits (Fig. 1). At $1.5 \mathrm{~h}$ after the cold stress, the frost-induced browning rates in the leaves were $0 \%$ in all the six accessions. At $3 \mathrm{~h}$, browning rate
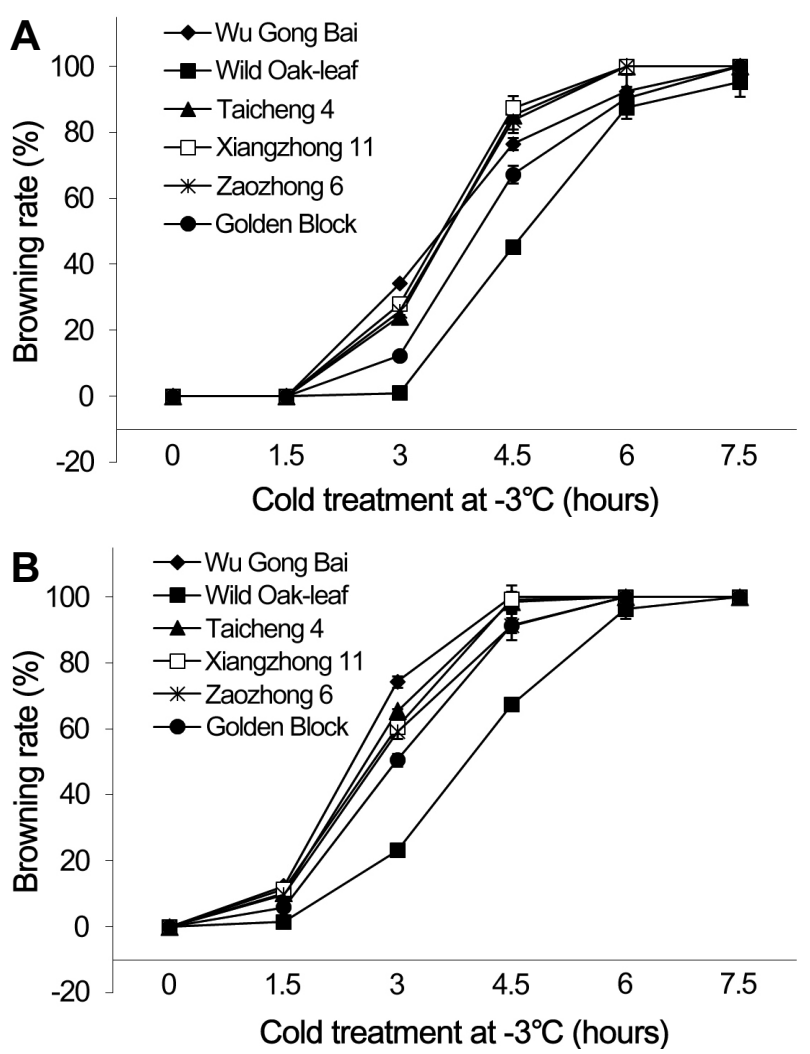

Fig. 1. Changes of browning rates in leaves (A) and fruits (B) of loquat accessions during cold treatment at $-3^{\circ} \mathrm{C}$. Bars represent standard error of the mean (three replicates). 
started to increase in the five commercial loquat accessions, while it remained at $0 \%$ in the 'Wild Oak-leaf' loquat. As the exposure time to cold stress increased to $4.5 \mathrm{~h}$, the browning rates increased dramatically. At $4.5 \mathrm{~h}$, the browning rate was only $45.3 \%$ in the 'Wild Oak-leaf' loquat, while it was over $65.0 \%$ in 'Golden Block', and more than $75.0 \%$ in 'Wu Gong Bai', 'Taicheng 4', 'Xiangzhong 11' and 'Zaozhong 6'. The increase in browning rate continued after $6 \mathrm{~h}$ of cold treatment and then to almost $100.0 \%$ damage at $7.5 \mathrm{~h}$.

Young fruits were more susceptible to cold injury than the leaves. Young fruits quickly developed frost damage symptoms (browning) after $1.5 \mathrm{~h}$ of cold treatment (Fig. $1)$, while no damage so far was found on the leaves at this stage. The wild relative 'Wild Oak-leaf' loquat was more cold tolerant than the commercial accessions. At 4.5 $\mathrm{h}$ after the cold treatment, the five commercial accessions had a browning rate over $90 \%$, while the browning rate was only about $67.4 \%$ in the 'Wild Oak-leaf' loquat.

\section{The changes in MDA content under cold stress}

There were significant differences in the leaf MDA content

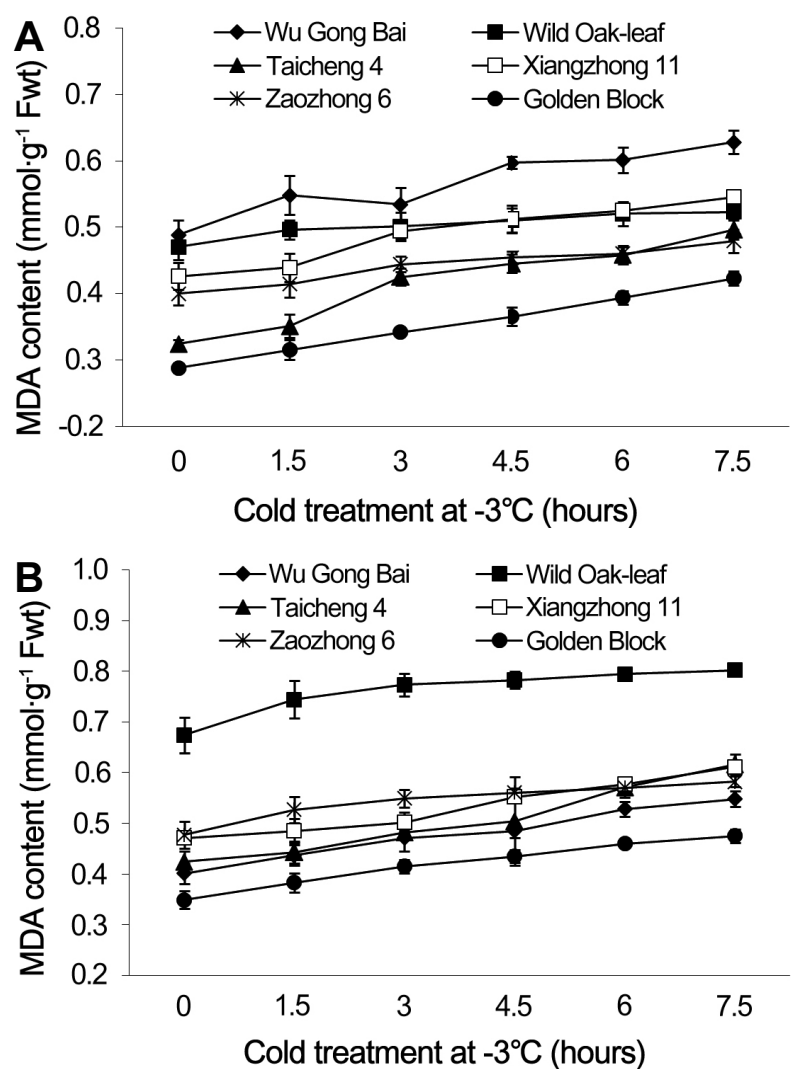

Fig. 2. Changes of MDA contents in leaves (A) and fruits (B) of loquat accessions during cold treatment at $-3^{\circ} \mathrm{C}$. Bars represent standard error of the mean (three replicates). between the accessions tested (Fig. 2), although the dynamic changes in MDA content had similar trending patterns in leaves and fruits. MDA content rose as the exposure time to cold stress increased. During the cold stress period from 0 to $7.5 \mathrm{~h}$ at $-3^{\circ} \mathrm{C}$, the MDA content in leaves increased by $11.3,19.8,27.9,28.7,46.9$, and $53.1 \%$ in 'Wild Oak-leaf' loquat, 'Zaozhong 6', 'Xiangzhong 11', 'Wu Gong Bai', 'Golden Block' and 'Taicheng 4', respectively. The change in MDA content was the greatest in 'Taicheng 4' and the least in 'Wild Oak-leaf' loquat, indicating that 'Wild Oak-leaf loquat was the most frost-tolerant among the accessions tested.

Similar results were obtained in the young fruits. The MDA content in the young fruits changed in an increasing order as 'Wild Oak-leaf' loquat (19.0\%), 'Zaozhong 6' (22.0\%), 'Xiangzhong 11' (29.7\%), 'Golden Block' (36.1\%), 'Wu Gong Bai' (36.7\%) and 'Taicheng 4' (45.3\%). The 'Wild Oak-leaf' loquat was again the strongest in frost-tolerance while 'Taicheng 4' was the least.

\section{The changes in POD and SOD activities under cold stress}

The changing trends of POD and SOD activities were
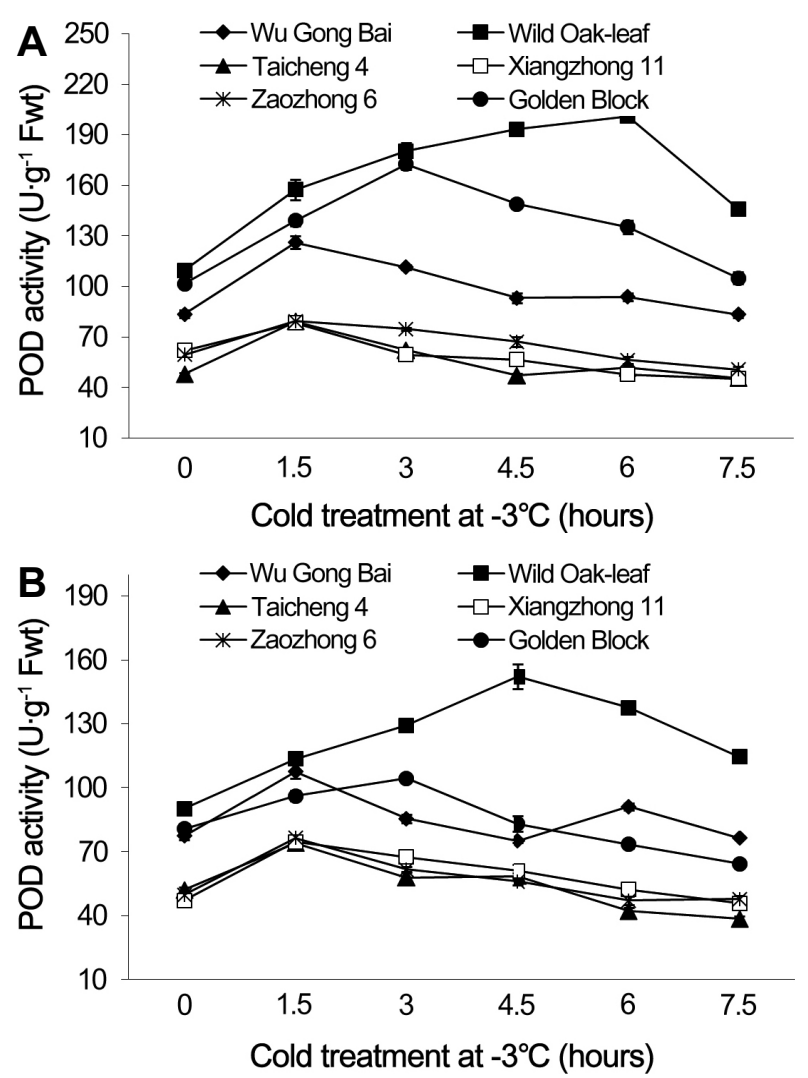

Fig. 3. Changes of POD activity in leaves (A) and fruits (B) of loquat accessions during cold treatment at $-3^{\circ} \mathrm{C}$. Bars represent standard error of the mean (three replicates). 
similar in the leaves and young fruits of the six loquat accessions under the cold stress at $-3^{\circ} \mathrm{C}$ (Figs. 3 and 4). The cold stress resulted in initial increases of POD and SOD activities, followed by decreases as the cold exposure time progresses. The POD and SOD activities in leaves and young fruits peaked between the $1.5 \mathrm{~h}$ and $6 \mathrm{~h}$ after the cold treatment, depending on the accessions. The POD and SOD activities in both leaves and young fruits peaked at $1.5 \mathrm{~h}$ after the cold treatment in 'Zaozhong 6', 'Xiangzhong 11', 'Wu Gong Bai' and 'Taicheng 4', while the 'Golden Block' peaked at $3 \mathrm{~h}$ and the 'Wild Oak-leaf' loquat peaked at $4.5 \mathrm{~h}$ for the young fruits and $6 \mathrm{~h}$ for the leaves. The 'Wild Oak-leaf' loquat had the highest levels of POD and SOD activities when compared to the five commercial accessions.

\section{The changes in CAT and APX activities under cold stress}

Under cold stress, the CAT and APX activities in the leaves and young fruit followed similar trending patterns in the six loquat accessions (Figs. 5 and 6). Cold stress
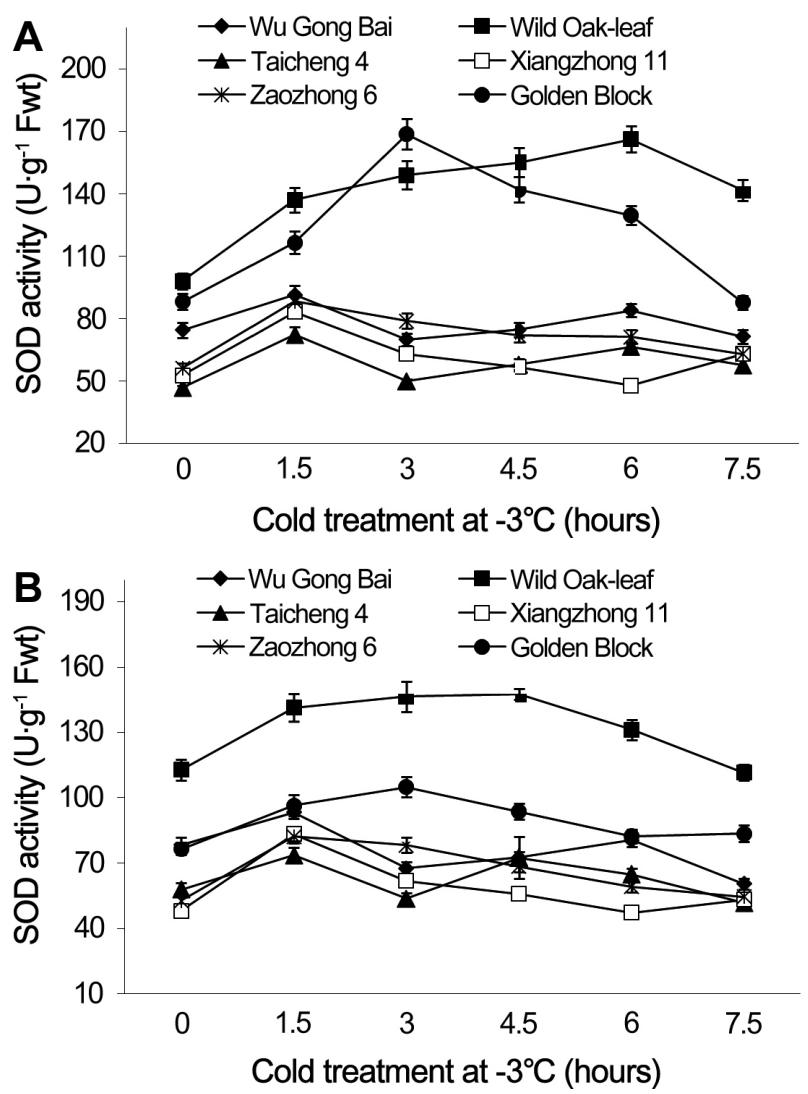

Fig. 4. Changes of SOD activity in leaves (A) and fruits (B) of loquat accessions during cold treatment at $-3^{\circ} \mathrm{C}$. Bars represent standard error of the mean (three replicates). induced an initial increase in enzymatic activities and then decreased as the time of cold stress prolonged.

Loquat accessions differed significantly in CAT activity in leaves. During the $7.5 \mathrm{~h}$ cold treatment period at $-3^{\circ} \mathrm{C}$, the value of 'Wu Gong Bai', 'Taicheng 4', 'Xiangzhong 11' and 'Zaozhong 6' plateaued at $1.5 \mathrm{~h}$, with an increase in the CAT activity by $53.3,27.3,13.4$, and $23.5 \%$, respectively, when compared with the $0 \mathrm{~h}$ treatment. The peak CAT value reached at $3 \mathrm{~h}$ and $6 \mathrm{~h}$ for 'Golden Block' and 'Wild Oak-leaf' loquat.

Similarly, the CAT activity in the young fruits of 'Wu Gong Bai', 'Taicheng 4', 'Xiangzhong 11' and 'Golden Block' peaked at $1.5 \mathrm{~h}$, and the its activity increased by 26.2 , $38.7,13.8$, and $27.6 \%$ respectively, when compared to the $0 \mathrm{~h}$ treatment. The peak value of 'Zaozhong 6' and 'Wild Oak-leaf' loquat occurred at $3 \mathrm{~h}$ and $6 \mathrm{~h}$, and their CAT activity increased by 38.8 and $30.4 \%$, respectively. The results showed that 'Wild Oak-leaf loquat was the strongest in frost tolerance, while there were no significant differences in frost tolerance between 'Taicheng 4', 'Xiangzhong 11'
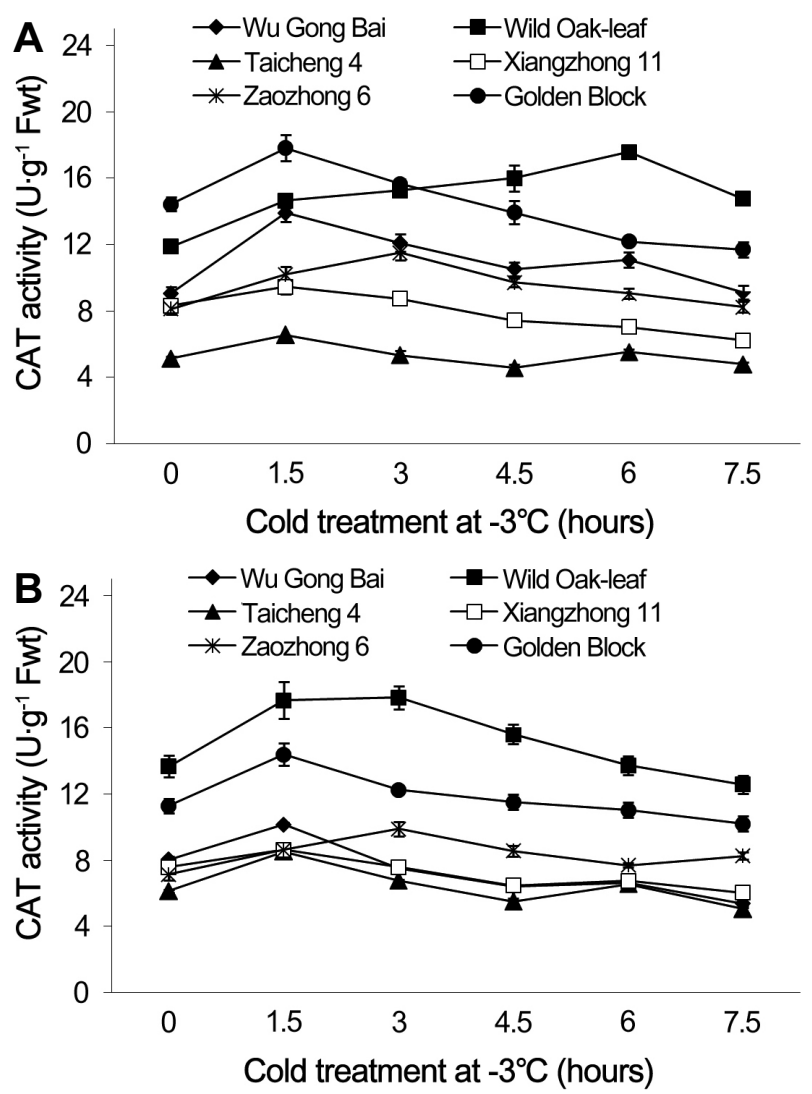

Fig. 5. Changes of CAT activity in leaves (A) and fruits (B) of loquat accessions during cold treatment at $-3^{\circ} \mathrm{C}$. Bars represent standard error of the mean (three replicates). 
and 'Zaozhong 6'.

During the various cold exposure under $-3^{\circ} \mathrm{C}$ stress, the APX activities in the leaves of 'Wu Gong Bai', 'Taicheng 4', 'Xiangzhong 11', 'Zaozhong 6' and 'Golden Block' reached maximum at $1.5 \mathrm{~h}$, and the APX activity increased by 46.6 ,

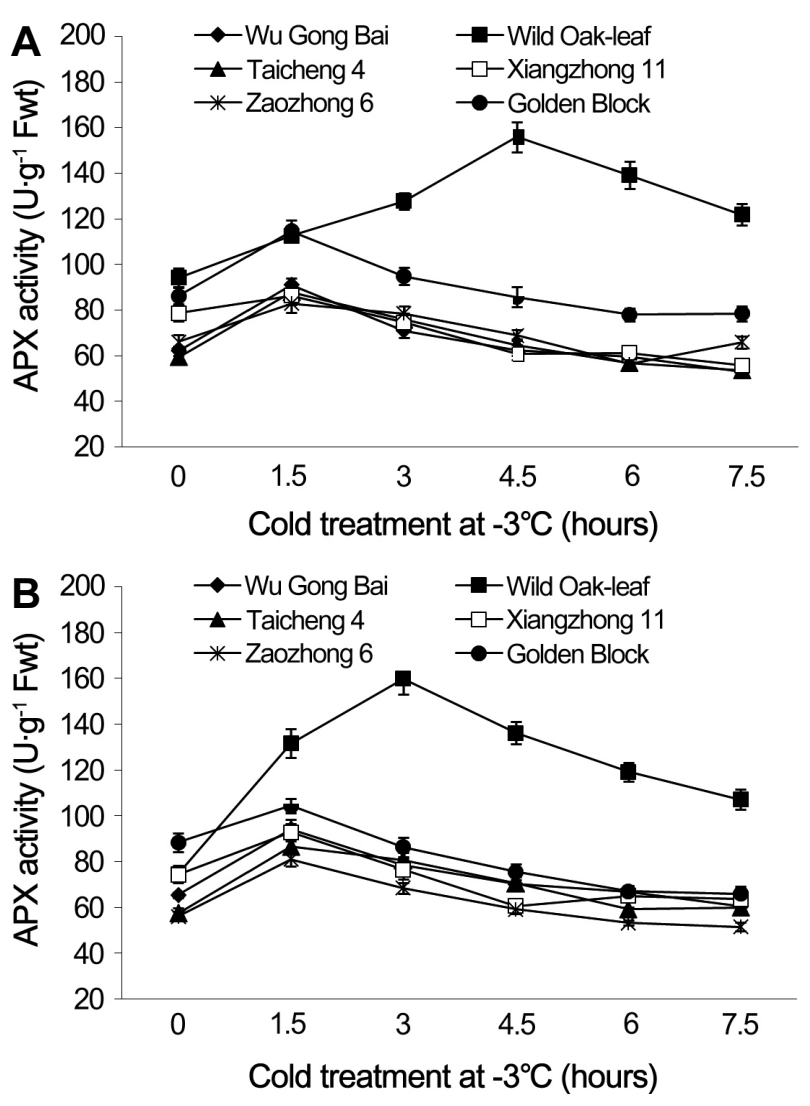

Fig. 6. Changes of APX activity in leaves (A) and fruits (B) of loquat accessions during cold treatment at $-3^{\circ} \mathrm{C}$. Bars represent standard error of the mean (three replicates).
$47.9,9.8,25.4$, and $32.8 \%$ respectively, when comparing to the $0 \mathrm{~h}$ treatment. The peak APX activity of 'Wild Oak-leaf' loquat occurred at $4.5 \mathrm{~h}$, with its value increased by $65.4 \%$.

The APX activity in the young fruits of 'Wu Gong Bai', 'Taicheng 4', 'Xiangzhong 11', 'Zaozhong 6' and 'Golden Block' peaked at $1.5 \mathrm{~h}$, and the APX activity increased by $44.0,50.8,25.0$, and $18.2 \%$ respectively, when comparing to the $0 \mathrm{~h}$ treatment, while the peak value of 'Wild Oak-leaf' loquat appeared at $3 \mathrm{~h}$ with an increase in the APX activity by $114.3 \%$.

\section{Frost tolerance and correlation in the leaves and young fruits}

There were significant correlations in MDA contents between the leaves and young fruits of all loquat accessions, with a correlation coefficient above 0.90 (Table 1). The value for 'Zaozhong 6', 'Golden Block' and 'Wild Oak-leaf' loquat were $0.96,0.99$ and 0.98 , respectively. The correlation coefficients of POD were significant in accessions Wu Gong Bai (0.85), Zaozhong 6 (0.93) and the 'Wild Oak-leaf' loquat (0.95). The SOD contents in the leaves were also significantly correlated with those in the young fruits of Wu Gong Bai (0.91), Xiangzhong 11 (0.96) and Zaozhong 6 (0.94). The CAT activities in the leaves and young fruits were significantly correlated in accessions Taicheng 4 (0.97), Xiangzhong 11 (0.96), Zaozhong 6 (0.90) and Golden Block (0.95). In addition, there were significant correlations in APX activity between the leaves and young fruits in all the accessions except the 'Wild Oak-leaf' loquat. In general, there was a good relasionship in MDA contents and enzyme activities between leaves and young fruits, with 21 out of 30 correlation coefficients being significant between the leaves and the young fruits.

Table 1. Correlation coefficients of MDA content and enzymatic activities between leaves and young fruits of different loquat accessions under cold stress.

\begin{tabular}{lcclcc}
\hline \multirow{2}{*}{ Loquat accessions } & MDA content & \multicolumn{4}{c}{ Enzyme activity } \\
\cline { 3 - 5 } & & POD & SOD & CAT & APX \\
\hline Wu Gong Bai & $0.93^{* * y}$ & $0.85^{*}$ & $0.91^{*}$ & 0.73 & $0.99^{* *}$ \\
Taicheng 4 & $0.93^{* *}$ & 0.81 & 0.68 & $0.97^{* *}$ & $0.96^{* *}$ \\
Xiangzhong 11 & $0.94^{* *}$ & 0.75 & $0.96^{* *}$ & $0.96^{* *}$ & $0.93^{* *}$ \\
Zaozhong 6 & $0.96^{* *}$ & $0.93^{* *}$ & $0.94^{* *}$ & $0.90^{*}$ & $0.91^{*}$ \\
Golden Block & $0.99^{* *}$ & 0.76 & 0.81 & $0.95^{* *}$ & $0.93^{* *}$ \\
Wild Oak-leaf loquat & $0.98^{* *}$ & $0.95^{* *}$ & 0.57 & 0.43 & 0.61 \\
\hline
\end{tabular}

${ }^{\mathrm{z}} \mathrm{MDA}=$ malondialdehyde, $\mathrm{SOD}=$ superoxide dismutase, $\mathrm{POD}=$ peroxidase, $\mathrm{CAT}=$ catalase and APX $=$ ascorbate peroxidase

${ }^{\mathrm{y}}$ Significant correlation: ${ }^{*} p<0.05 ;{ }^{* *} p<0.01$. 


\section{Discussions}

A previous study has showed that critical temperature of cold injury for Zaozhong 6 was $-3^{\circ} \mathrm{C}$ (Xie and Li, 2006). The present study demonstrated that a short period (< $1.5 \mathrm{~h}$ ) of cold stress at $-3^{\circ} \mathrm{C}$ did not cause significant injury to the six loquat accessions, with no browning for the leaves, and a browning rate of less than $10 \%$ for the young fruits. In addition, direct observation showed that 'Wild Oak-leaf loquat was the strongest in frost-tolerance, followed by 'Golden Block'. The 'Wu Gong Bai', 'Taicheng 4', 'Xiangzhong 11 ' and 'Zaozhong 6' were relatively weak in frost-tolerance. The young fruits were more sensitive to cold damage than the leaves.

MDA content is an important lipid peroxidation marker to assess cold injury, as it increases when membrane permeability rises as a result of increased peroxidation of membrane under cold stress. Sun et al. (2011) reported that a lower level of MDA was associated with frost tolerance. Our results from both leaves and young fruits showed that the MDA content increased steadily as the cold treatment prolonged. However, MDA content had the least change in 'Wild Oakleaf' loquat, indicating that it was the most frost-tolerant, as compared to 'Taicheng 4', 'Xiangzhong 11' and 'Zaozhong 6'. Therefore, the relative change in MDA contents before and after cold treatment could be a useful biomarker for loquat frost tolerance.

The reactive oxygen species induced under plant stress are harmful to plant cells (Wang et al., 1989). Some enzyme systems in the plants can reduce the negative impact of these reactive oxygen species. Commonly all these enzymes are in a dynamic balance under normal conditions (Duan et al., 1998). A short period of cold stress treatment will lead to increased enzymatic activity and decreased active oxygen content, while an extended period of cold treatment will lead to decreased enzymatic activity and increased reactive oxygen content (Lin et al., 2001). The present study showed that the activities of POD, SOD, CAT and APX in loquat leaves and young fruits increased during the first $1.5 \mathrm{~h}$ of cold stress, suggesting that a short period of cold treatment could be used as an effective method for loquat acclimation to cold temperatures. However, the activities of all enzymes then decreased as the exposure time progressed, which would be harmful to loquat cells and caused the injury in leaves and fruits.

These oxygen-scavenging enzymes have been known to be associated with tolerance to external stresses, such as salinity, heat, drought and coldness in plants (Heidari et al., 2012). It was reported that high salinity tolerance of wheat cultivars was associated with higher activities of antioxidant enzymes, increased accumulation of proline, and reduced levels of $\mathrm{H}_{2} \mathrm{O}_{2}$ as compared to the sensitive cultivar (Hassanpour et al., 2012). Similar relationships were also reported under cold stress (Xuan et al., 2013)

Yue et al. (2010) and Liu et al. (2007) reported that activities of antioxidant enzymes were different depending on plant cultivars. The activities of POD, SOD, CAT and APX in 'Wild Oak-leaf' loquat increased steadily even at $4.5 \mathrm{~h}$ under $-3^{\circ} \mathrm{C}$ stress, while the enzymatic activities in the other five accessions trended to decrease over time. In addition, the enzymatic peak value in strongly frosttolerant accessions appeared later than that in the weakly tolerant accessions.

It showed that the changes in the enzymatic activities of different reactive-oxygen-scavenging enzymes in loquat tested were not all consistent with the changes in cold injury during the cold treatment. Therefore, the frost tolerance in loquat can't be relied on any single enzyme activity; it relies on the use of multiple enzyme activities.

There was a close correlation in MDA content and enzymatic activities between the leaves and young fruits in the 5 commercial loquat accessions. For the 'wild Oak-leaf loquat, MDA content and POD activities in leaves were also highly correlated with these enzymatic activities in the young fruit. The frost tolerance in young fruit could therefore be predicted from that in the leaves. This study has demonstrated that some biochemical enzyme markers could be useful in identifying the frost tolerance in loquat. These markers could assist in speeding up the selection process for breeding frost tolerant loquat. However, the number of loquat accessions in this study was limited. Further research is needed to study these biochemical markers with a large collection of loquat germplasm, and mathematical modeling approach could be used to integrate all the enzymatic data to select most relevant biochemical index for predicting the frost tolerance in loquat.

\section{Literature Cited}

Chen, L.S. and L. Cheng. 2003. Both xanthophyll cycle-dependent thermal dissipation and the antioxidant system are up-regulated in grape (Vitis labrusca L. cv. Concord) leaves in response to $\mathrm{N}$ limitation. J. Exp. Bot. 54:2165-2175.

Duan, Y., S. Song, and J. Fu. 1998. Effects of calcium on senescence in detached leaves of hybrid rice. Acta Sci. Nat. Univ. Sunyatseni 37:83-87.

Ferreres, F., D. Gomes, P. Valentão, R. Gonçalves, R. Pio, E.A. Chagas, R.M. Seabra, and P.B. Andrade. 2009. Improved loquat (Eriobotrya japonica Lindl.) cultivars: Variation of phenolics 
and antioxidative potential. Food Chem. 114:1019-1027.

Giannopolitics, C.N. and S.K. Ries. 1977. Superoxide dismutase. 1.Occurrence in higher plants. Plant Physiol. 59:309-314.

Hassanpour, H., R.A. Khavari-Nejad, V. Niknam, F. Najafi, and K. Razavi. 2012. Effects of penconazole and water deficit stress on physiological and antioxidative responses in pennyroyal (Mentha pulegium L.). Acta Physiol. Plant. 34:1537-1549.

Heidari, B., M. Pessarakli, A. Dadkhodaie, and N. Daneshnia. 2012. Reactive oxygen species-mediated functions in plants under environmental stresses. J. Agric. Sci. Technol. B2:159-168.

He, Q., X.W. Li, G.L. Liang, K. Ji, Q.G. Guo, W.M. Yuan, G.Z. Zhou, K.S. Chen, W.E. van de Weg, and Z.S. Gao. 2011. Genetic diversity and identity of Chinese loquat cultivars/accessions (Eriobotrya japonica) using apple SSR markers. Plant Mol. Biol. Rep. 29:197-208.

Hueso, J.J. and J. Cuevas. 2008. Loquat as a crop model for successful deficit irrigation. Irrigation Sci. 26:269-276.

Jiang, R.F., Y.F. Chen, and Y.Q. Lin. 2010. Feature analysis and forecast research of loquat frost injury in Putian. Sci. Technol. West China 9:6-8.

Jiang, W.B., Y.L. Wang, and K. Ma. 1992. Effect of osmo protective substances on cold hardiness of fig. Acta Hortic. Sin. 19:371-372.

Lin, D., Y.J. Yang, W.L. Fan, and Y.T. Wei. 2001. Effects of low temperature on active oxyen metabolism of tomato seedlings leaves. Liaoning Agric. Sci. 5:5-8.

Liu, H.X., S.X. Zeng, Y.R. Wang, P. Li, D.F. Chen, and J.Y. Guo. 1985. The effect of low temperature on superoxide dismutase in various organelles of cucumber seedling cotyledon with different cold tolerance. Acta Phytophysiologica Sinica 11:48-57.

Liu, X.Q., X.F. Wang, and Y.J. Piao. 2007. A Study on cold tolerance of different phalaenopsis cultivars. Acta Hortic. Sin. 34: 425-430.

Liu, Z.Q. and S.C. Zhang. 1994. Plant resistant physiology, p. 371-372. China Agriculture Press, Beijing, China.

Peng, C.C. and Z.H. Sun. 2000. Changes of SOD and CAT activities of citrus protoplast during cold acclimation. J. Huazhong Agric. Uni. 19:384-387.

Rao, M.V., G. Paliyath, and D.P. Ormrod. 1996. Ultraviolet-B-and ozone-induced biochemical changes in antioxidant enzymes of Arabidopsis thaliana. Plant Biol. 110:125-136.

Ren, H.H., W. Huang, and F.M. Zhang. 2002. Effects of low temperature and poor light on some physiological parameters of tomato. J. China Agric. Uni. 7:95-101.

Sun, S.X., J. Li, M.Y. Tu, D. Chen, H.J. Xie, and G.L. Jiang. 2011. Freezing damage factors and physiological indexes of frost resistance in loquat. ISHS Acta Hortic. 887:171-176.

Wang, F., H. Wang, D.W. Chen, and J.R. Li. 1999. A Study on the hardiness of flower organ of Apricot accessions. Acta Hortic. Sin. 26:356-359.

Wang, H., J.P. Peng, S.M. Fang, G.D. Zheng, C.J. Qiu, Q. Huang, Y.S, Chen, and G.H. Zheng. 2008. Relationship between ice nucleation bacteria and frost in loquat. Plant Prot. 34:43-46.

Wang, J.H., H.X. Liu., and T. Xu. 1989. The role of superoxide dismutase (SOD) in stress physiology and senescence physiology of plant. Plant Physiol. Comm. 1:1-7.

Xie, Z. and J. Li. 2006. Definition on Freezing Injury Temperature of 'Zaozhong 6' Loquat Young Fruits and Division of Suitable Cultivating Area. Fujian Fruits 1:7-11.

Xuan, J., H. Zhang, and Z. Guo. 2013. Physiological response of zoysiagrass in urban landscapes to low temperature stress. ISHS Acta Hortic. 999:253-261.

Yang, F.J., B.J. Li, and Y.G. Gao. 2003. Recent progress in the study of cold resistance of fruit tree. J. Heilongjiang Aug. First Land Reclam. Univ. 15:23-29 15:23-29.

Yue, H., G.H. Li, G.W. Li, L.L. Chen, G.H. Kong, and G.P. Liang. 2010. Studies on cold resistance of different macadamia cultivars. Acta Hortic. Sin. 37:31-38.

Zhou, B., J. Chen, Z. Ji, and R. Cai. 1999. Changes of superoxide dismutase activity and water-soluble protein content in bananas during winter. J. Fruit Sci. 16:192-196.

Zhou, Z. and G. Lu. 1994. The effect of chilling stress on membrane lipid peroxidation and protective enzyme in pepper seedlings. Acta Agric. Boreali-Occidentalis Sin. 3:51-56.

Zhu, G.L. 1990. Plant Physiology Experiment, p. 38-39. Beijing University Press, Beijing, China. 